\title{
Uthatyatho nxaxheba luluntu: Ingaba INkundla yoMgaqo siSeko ilunike ntoni uluntu?
}

Merafong Demarcation Forum kunye naBanye v UMongameli woMzantsi Afrika kunye naBanye [2008] ZACC 10

LINDA NYATI

Umfundi weB Comm. (Law) kwiDyunivesithi yaseNtshona Koloni

\section{INTSHAYELELO}

IFreedom Charter ${ }^{1}$ yachaza kwiminyaka engaphambili: "Abantu baya kulawula". Xa abaqulunqi boMgaqo siSeko babeseka inkqubo yokwenziwa kwemithetho oko bekwenzela idemokhrasi yethu, kusenokwenzeka ukuba ezingqondweni zabo babe nale ngcamango. Idemokhrasi esekelezelwe kuMgaqo siSeko yoMzantsi Afrika ubume bayo buqulathe ukumela kunye nokuba yinxalenye; isolotya lokumela liquka idemokhrasi ebandakanya onke amaqela ezopolitiko, le meko isenzeka ngokuthi kusoloko kubakho unyulo olusekelezelwe kuluhlu lukawonke-wonke lwabavoti kunye nomelo ngokwenani lwevoti²; le nkqubo yokubandanyakeka ayipheleli ekuthabatheni inxaxheba ekuvoteni ukuphela rhoqo emva kweminyaka emihlanu, kuloko ithi iqinisekise ukuba umhlali ngamnye weli lizwe unendima ayidlalayo kubomi beli lizwe ngelixa unyulo lungekafiki. Ezi nkalo zimbini akufuneki zithatyathwe njengezo zikhabanayo kodwa zithatyathwe njengezo zifezekisanayo. Kungokoke uthabatho nxaxheba loluntu luthi lubandakanywe nelungelo lokuthatha inxaxheba kwezopolitiko. Ilungelo lokuthatha inxaxheba kwezopolitiko phantsi koMthetho weHlabathi ${ }^{3}$ othi uvumelane nelungelo lokuthatha inxaxheba kwizinto ezenzeka kubomi boluntu.

1 IFreedom Charter eyamkelwa kwiNkongolo yoLuntu, eKliptown ngo 1955.

2 Motala kunye noRamaphosa, 'Constitutional Law: Analysis and cases' Oxford University Press (2002) 9.

3 UMzantsi Afrika wamkela isiVumelwano seHlabathi esimayela namaLungelo oLuntu ezoPolitiko nge3 Okthobha 1994 kwaye wawuphumeza nge10 Disemba 1998.

Inqaku 25 lifundeka olu hlobo:

Ummi ngamnye weli lizwe uya kuba nelungelo kunye nethuba ngokungaphandle kwezo ngcamango zichongwe kwinqaku 2 kwaye engasayi kuba nezithintelo ezingacingisiswanga:

(a) ukuthatha inxaxheba kwimibandela yoluntu oko ekwenza ngqo okanye oko kusenziwa ngbameli abakhethwe ngokukhululekileyo

(b) avote kwaye abe nokunyulwa xa kuqhubeka uvoto oluya kuba lolungenamkhethe kuwonkewonke kwaye oluya kuqhubeka ngokufihlakeleyo, luqinisekisa ingcamango ezikhululekileyo zemfuno zabavoti." 
Ngamanye amazwi uthatyatho nxaxheba luluntu kwinkqubo yoqulunqo lwemithetho luyinxalenye yedemokhrasi. KuMgaqo siSeko woMzantsi Afrika $^{4}$ (uMgaqo siSeko") ilungelo lokuvota ${ }^{5}$ xa kuvotwa liqinisekisa ukuba urhulumente onyulwe ngenkqubo yedemokhrasi kumele ukuba abe ngulowo ukwaziyo ukumela izenzo zakhe ${ }^{6}$, akwazi ukuphendula kwaye angafihli nto ${ }^{7}$. UMgaqo siSeko ukwachaza ukuba iNdibano yeSizwe eyonyulwe luluntu, kumele ukuba uqinisekise "urhulumente woluntu phantsi koMgaqo siSeko ...ngokuthi idale iqonga likazwelonke apho imibandela yoluntu inokuphononongwa khona"8. Kukumacandelo 59(1)(a), 72(1)(a) kunye no-118(1)(a) ${ }^{9}$ woMgaqo siSeko athe aqulunqa uthatho nxaxheba koluntu kule nkqubo yoqulunqo lwemithetho kazwelonke. Xa uluntu lukwazi ukuthatha inxaxheba kuqulunqo lwemithetho kazwelonke, umntu ezenzela buqu okanye ekwenza ezibandakanya nabanye abantu, oko kungumqondiso wokuba nabani na uthatyathwa njengobalulekileyo kwaye nengcamango zakhe zithi zisiwe iso ngurhulumente. ${ }^{10}$

INkundla yoMgaqo siSeko ("INkundla") ithe kumaxa angaphambili yacelwa ukuba iphonononge kwaye icacise lendima ekumele idlalwe "ukuququzelela uthatyatho nxaxheba koluntu" ekusekweni kwemithetho nezinye inkqubo ezisekelezelwe kumaqumrhu anyulwe ngokusemthethweni oko kusenziwa phantsi koMgaqo siSeko. Kutsha nje iNkundla kuye kwafuneka ichophele lombandela kwityala leMerafong Demarcation Forum kunye naBanye $v$ UMongameli woMzantsi Afrika kunye naBanye. ${ }^{11}$

Eli nqaku lifuna ukuphonononga ukuba ingaba yintoni intsingiselo yothatyatho nxaxheba luluntu kuqulunqo lwemithetho olukhuselwe kuMgaqo siSeko, ingakumbi xa kujongwa ityala laseMerafong, nophengululo ngokusemthethweni kwale meko ziinkundla.

4 Umthetho we108 ka1996

5 Icandelo 19 (2) lomGaqo siSeko liqulathe oku:

-Wonke ummi weli lizwe unelungelo lokuvotela naliphina iqumrhu elisekelezelwe phantsi koMgaqo siSeko oko ekwenza ngokukhululekileyo nangokusulungekileyo kwaye kusenzeka rhoqo ,

6 Icandelo 41 (1) (c) loMgaqo siSeko liqulathe oku:

-Onke amacandelo karhulumente nazo zonke izintlu zaseburhumenteni nganye nganye kumele zibe nakho ukuzisa uburhulumente obusebenzayo, obukwaziyo ukumela izenzo zazo kwaye obungafihlisi nto oko lukwenzela abemi boMzantsi Afrika uphela.

7 Enye yeenqobo kwisiseko esiqulathwe kuMgaqo siSeko ukhankanyiwe kwicandelo 1 (d) njengelungelo lokuvota, umqulu oqulathe uluhlu lwabavoti beli lizwe, ukubanjwa kolonyulo rhoqo kunye nentlanganisela ebandakanya amaqela onke ezopolitiko kurhulumente wedemokhrasi, kuqinisekiswe nokumela izenzo zakho, ukukwazi ukuphendula kunye nokungafihlisi.

8 Icandelo 42 (3):

-iNdibano yeSizwe ityunjwa ukuba imele abantu kwaye iqinisekise urhulumente wabantu phantsi koMgaqo siSeko. Ikwenza oko ngokuthi inyule uMongameli, kwaye yenze kubekho iqonga likawonke-wonke lesizwe eliya kuqwalasela imicimbi yoluntu....

9 Icandelo 59(1)(a) INdlu yoWiso-mthetho kumele- (a) iququzelele uthatyatho nxaxheba koluntu kuqulunqo lwemithetho kunye nezinye inkqubo zepalamente neekomiti zayo; ...

Icandelo 72(1)(a)IBhunga lamaPhondo leSizwe kumele- (a) liququzelele uthatyatho nxaxheba koluntu kuqulunqo lwemithetho kunye nezinye inkqubo zepalamente neekomiti zayo; ...

Icandelo 118(1)(a) Ipalamente yephondo kumele- (a) iququzelele uthatyatho nxaxheba koluntu kuqulunqo lwemithetho kunye nezinye inkqubo zepalamente neekomiti zayo; ...

10 URhulumente v Makwanyane 1995 (6) BCLR 665 (CC), ngokuka O’Regan iJaji, umhlathi 329.

11 [2008] ZACC 10. 


\section{INDIMA EDLALWE NGUMGAQO SISEKO KUTHATYATHO NXAXHEBA LULUNTU}

Korhulumente bangaphambili uninzi lwabemi boMzantsi Afrika babe khutshelwe ngaphandle kubomi boluntu jikelele ${ }^{12}$ ingakumbi ukuvinjwa kwabo ilungelo lokuvota. Ekwahlukaneni nondebe-ndala, amacandelo 59(1)(a), 72(1) (a) kunye no-118(1)(a) ${ }^{13}$ aba nesiphakamiso kwiNdlu yoWiso-mthetho yeSizwe, kwiBhunga lamaPhondo leSizwe kunye namaBhunga amaPhondo aqulunqa imithetho, ukuba aququzelele uthatyatho nxaxheba luluntu xa esenza iinkqubo zawo ezinxamnye nemithetho, konke oko kusekelezelwe kuMgaqo siSeko.

Nangona amaqumrhu ajongene nemithetho enelungelo lokuqulunqa iinkqubo ema yilandelwe xa kuqulunqwa uthatyatho nxaxheba luluntu, iNkundla yoMgaqo siSeko yaye yabeka ummiselo kwityala leDoctors for Life International $v$ USomlomo weNdlu yoWiso-mthetho yeSizwe ${ }^{14}$. Imibandela eyabe iphambi kweNkundla kwityala leDoctors for Life International yabe iyile (i) iyintoni indima ekumele ukuba yenziwe ukuququzelela uthatyatho nxaxheba luluntu; (ii) ingaba ipalamente iwanikezele kusini na amandla ayo ekubeni iququzelele uthatyatho nxaxheba luluntu kwinkqubo zokuyilwa kwemithetho kazwelonke ebandakanya imithetho ethile yempilo; kunye (iii) siyintoni isiphumo sokuba semthethweni komthetho ukubangaba uqulunqo lokubandakanya uluntu kuthatho nxaxheba beluneziphene. Uvavanyo olubekiweyo lolo luqwalasela ukuba ingaba ipalamente yenze ngokufanelekileyo na ukudlulisela imfanelo zokubandakanya uthatyatho nxaxheba luluntu. Ezi ngongoma zilandelayo ziya kuthi zininikwe ingqwalasela ukufumanisa ezi nkathalo: (i) inkangeleko yalo mthetho kujongwe kuwo; (ii) ukubaluleka kwalo mthetho; (iii) igalelo lawo kuluntu ${ }^{15}$ kunye neminye imibandela eya kuthi ixhomekeke kwimeko zetyala ngalinye. Ngaphezu koko, noko angamabini amasolotya abandakanywa luxanduva lokuqulunqa uthatyatho nxalenye luluntu; okokuqala, ukuveza amathuba okuthatha inxaxheba koluntu kwinkqubo yokuseka imithetho, okwesibini, ukuqinisekisa ukuba uluntu lunakho ukuthatha elo thuba lululinikiweyo. ${ }^{16}$ USachs iJaji, evumelana nesigqibo sesininzi, wagxininisa "kwintsingiselo ebalulekileyo"17 yothatyatho nxaxheba luluntu kule demokhrasi yethu kwaye wathi igalelo lokubandakanywa koluntu kufuneka luqulathe oku:"

"Onke amaqela anomdla kumthetho kufuneka azive ukuba ebe elinikiwe ithuba elililo lokuveza izimvo zalo, kwaye lanikwa inkathalo njengabemi beli lizwe, kwaye izimvo zalo zilulutho kwaye ziya kuthi zinikwe ingqwalasela ngelixa zinakho ukuba zinganefuthe elingundoqo kwizigqibo ezithile. Injongo zezo zisekelezisiweyo kwakunye nezo zenziwayo: Abantu ababandanyekayo kumele banikwe imbeko ebafaneleyo njengabemi beli lizwe abanenkathalo, kwaye abaqulunqi mithetho kumele babe nazo zonke iziphakamiso eziya kuthi zibenze babe nakho owona mthetho unguwo". ${ }^{18}$

12 Brink $v$ Kitshoff 1996 (6) BCLR 752 (CC) kumhlathi 40.

13 Jonga u9 ngasentla.

14 Doctors for Life International v USomlomo weNdlu yoWiso-mthetho yeSizwe kunye naBanye 2006 (12) BCLR 1399 (CC).

15 Doctors for Life International (fn 14 ngasentla) kumhlathi 129.

16 Doctors for Life International (fn 14 ngasentla) kumhlathi 129 . .

17 Doctors for Life International (fn 14 ngasentla) ngokukaSachs, umhlathi 226.

18 Doctors for Life International (fn 14 ngasentla) ngokukaSachs, umhlathi 235. 
Esi sigqibo siphume ecaleni kwimbali yangaphambili ebe isithi ibonakalise izigqibo ezingangqamaniyo zemithetho kwakunye nokujongelwa phantsi koninzi loluntu loMzantsi Afrika. Lo nto yenza nomfuziselo wokuba iNkundla yoMgaqo siSeko izimisele ekujongeni "iimeko zohlukumezo zangaphambili" ingakumbi nokuba ipalamente kumele ukuba izimele izenzo zayo kwabo bantu bathe banyulwa ngabo. IDoctors for Life iye yabeka umkhomba-ndlela woMgaqo siSeko onika uxanduva lokubandakanya inxalenye yoluntu kodwa okona kuqondisisa kukuko koko kuye kwayilwa mva-nje yinkqubo yomthetho. Kungalityalwanga ezingqondweni imbali yoMzantsi Afrika, umntu unokuba nethemba lokhuseleko phantsi koMgaqo siSeko elikhusela ukuphunyezwa ngokungangqamaniyo kwemithetho.

\section{UTHATYATHO NXAXHEBA LULUNTU KUGUQULO LWEMIDA YEPHONDO}

UMzantsi Afrika unembali yokufuduswa kwabantu ngenkani, nolawulo lwentshukumo phantsi kooZimele-geqe njenge nzame zokwandisa umsantsa phakathi kwabo bangenanto kunye nabo bazizityebi, abasezidolophini kunye nabo basemaphandleni, naphakathi kwentlanga kunye naphakathi kwabemi beli lizwe. Ekuqondweni kokuba ulawulo lomasipala aba mida ixananazileyo isekelezwe zingxaki ${ }^{19}$, urhulumente kwakumele xa eyiphelisa achule ukunyathela kuba kusenokwenyuka imisindo (njengoko uluntu loMasipala waseMerafong lubonakalisa) ukubangaba abantu banembono yokuba abakhange babandakanywe ngokufaneleyo njengoko kwakusakwenzeka kwimthetho yocalu-calulo njenge Group Areas $\mathrm{Act}^{20}$.

Kwityala loMasipala waseMatatiele kunye naBanye v UMongameli woMzantsi Afrika naBanye ${ }^{21}$ umba woMgaqo siSeko yayikukuba ingaba yalandelwa ngokufanelekileyo na inkqubo ngelixa kuphunyezwa uHlalutyo lweShumi elineSibini loMgaqo siSeko ka2005 (uHlalutyo lweShumi elineSibini) owawuza kuba negalelo ekuguquleni umda wephondo leKwaZulu-Natala kunye neleMpuma Koloni. AbaBheni bacela-umngeni kubuGaqo siseko boHlalutyo lweShumi elineSibini kwakunye nalowo Mthetho woMasipala weMida eXananazileyo kunye nowokuRhoxisa iMiba Eyelele Apho we23 ka 2005 besithi iNdlu yowiso-mthetho yePhondo leKwaZulu-Natala ayikwazanga ukwenza umsebenzi wokuqulunqa uthatyatho nxalenye luluntu kwaye ngoko ke lemithetho zange ibe nakho ukuphunyezwa njengokwe zindululo zoMgaqo siSeko. ${ }^{22}$

19 Isaziso saseburhulumenteni esibhekiswa kwiPresident's Coordinating Council, 1 Novemba 2002: ULawulo lwemida loMasipala exananazileyo.

20 Umthetho we41 ka 1950.

21 UMasipala waseMatatiele kunye naBanye v UMongameli woMzantsi Afrika naBanye Matatiele 2006 ZACC 12, Matatiele 2.

22 Icandelo 74(3)(b)(ii)-

"(3) Kunye nasiphina isindululo soMgaqo siSeko sinakho ukuhlaziywa ngoMthetho oYilwayo onokuphunyezwa-

(a) yiNdibano yeSizwe, oko kuxhaswa yivoti yesibini kwisithathu yamalungu ale ndlu; kunye

(b) neBhunga lamaPhondo leSizwe elixhaswa yivoti yamaphondo amathandathu, ukubangaba uhlalutyo-

(ii) luguqula imida yamaphondo, ubungangamsha, imisebenzi okanye iindawo;... 
Icandelo 74(3)(b)(ii) lithi libeke inkqubo ekumele ilandelwe xa kuguqulwa imida yamaphondo. IziNdlu zowiso-mthetho zamaphondo (okanye yephondo) abandakanyekayo kumele i(zi)phumeze lo mthetho uyilwayo okanye inxenye yalowo uhlonyelweyo othi ubandakanye elo phondo ${ }^{23}$; oko kunika elo phondo libandanyekayo ithuba lokukhusela imida yalo ngemfezeko. ${ }^{24}$ Nanjengoko la maphondo mabini abandakanyekayo, iNdlu yowiso-mthetho yaseKwaZulu-Natala kunye neyeMpuma Koloni kumele zilandele icandelo 74(8), iKwaZulu-Natala ayithathanga manyathelo okubandakanya uluntu kwizigqibo zayo zokuxhasa uguqulo lwemida. Kwisigqibo sayo iNkundla yathi:

"[i]Ndlu yowiso-mthetho yaseKwaZulu-Natala yayifanele ukuphumeza elo solotya loHlalutyo loMthetho weShumi elineSibini elithi lidlulise indawo ebisakuba yinxalenye kaMasipala wesithili saseMatatiele iyidlulisela ukusukela kwiphohondo leKwaZuluNatala ukya kwelaseMpuma Koloni. UMgaqo siSeko ucingela ukuba ukuvunyelwa mayela neCandelo 74(8) liya kunikwa yipalamente yephondo ebandanyekayo oko kusenzeka emva kokuba ihambisene kunye nezindululo ezibonelelwe kwicandelo 118(1) (a)" ${ }^{25}$ (apho ndigxininisa khona)

Ukususela kwesi sicatshulwa singasentla kucacile ukuba kutheni na inkundla, xa yayisebenzisa uvavanyo lweDoctors for Life International, yafumanisa ukuba isigqibo sepalamente yephondo asinakusebenza. ${ }^{26} \mathrm{Oku}-$ nokufundwa kweli tyala kukuba ukuhambisisana kunye nezindululo ezithi zibangele uthatyatho nxaxheba luluntu kuyimfuneko kwinkqubo yokubunjwa kwemithetho, ingakumbi xa umthetho ubhekiselele kumba obaluleke njengo kuguqulo lwemida yamaphondo.

\section{IMERAFONG DEMARCATION FORUM KUNYE NABANYE V UMONGAMELI WOMZANTSI AFRICA KUNYE NABANYE ${ }^{27}$}

Kwinzame zokuzama ukupheliswa komasipala aba mida exananazileyo mibini imithetho eyayilwayo ${ }^{28}$ eyathi yaphunyezwa kwiNdlu yowiso-mthetho yeSizwe kwaye ngokubhekiselele kwicandelo 74 inkqubo yoseko mithetho bekumele ukuba igqityezelwe kwiBhunga lamaPhondo leSizwe. Oku kwachazwa mayela noMasipala waseMerafong njengomzekelo waba masipala:

"UMasipala weSithili saseMerafong uya kuthi ungabandakanywa kuMasipala wommandla weWest Rand eGauteng ukuze ubandakanywe kwingingqi yoMasipala

23 Umthetho 74(8) woMgaqo siSeko;

Ukubangaba umthetho oyilwayo ochazwayo kwisihlokwana (3) (b), okanye nayiphina indawo eyinxenye yalo mthetho uyilwayo ubandakanya naliphina iphondo okanye amaphondo, iBhunga lamaPhondo leSizwe alinakho ukuphumeza umthetho oyilwayo okanye elo solotya lingusingaye ngaphandle kokuba uye waphunyezwa yipalamente okanye ipalamente zamaphondo.

24 Ex parte Chairperson of the Constitutional Assembly: In re Certification of the Constitution of the Republic of South Africa, 1996 (4) SA 744 (CC); 1996 (10) BCLR 1253 (CC) umhlathi 233.

25 Matatiele 2 (fn 21 ngasentla) umhlathi 85.

26 Matatiele 2 (fn 21 ngasentla) umhlathi 89.

27 Jonga u11 ngasentla.

28 uHlalutyo lweShumi elineSibini loMgaqo siSeko ka2005 kunye nomthetho woMasipala weMida eXananazileyo kunye nowokuRhoxisa iMiba Eyelele Apho we23 ka 2005. 
weSouthern District eMntla Ntshona. IWestonaria iya kuthi ihlale iphantsi koMasipala wommandla weWest Rand". 29

Uhambelana nezindululo zoMgaqo siSeko kwaba yimfuneko ukuba kuqulunqwe uthatyatho nxaxheba luluntu iphantsi kwecandelo 118(1)(a) mayelana nale mithetho.

Kwahambeka ngolu hlobo: INdlu yowiso-mthetho yePhondo laseGauteng, ithumele iKomiti yeMicimbi yeSebe, kunye neyePhondo loMntla Ntshona aye abandakanya uluntu lwaseMerafong ngale mibandela apho amaqela oluntu avakalisa ukuchasana nokubandakanywa kwawo phantsi kwePhondo loMntla Ntshona. Kule ndibano iKomiti yePhondo laseGauteng yagqiba ukuba ivumelane noluntu ngokuthi ivumela ukupasiswa kwalemithetho ukuba isolotya elithi iMerafong izakubandakanywa eMntla Ntshona liyasuswa.

Emveni koko, iKomiti yeMicimbi yeSebe yadibana nabacebisi bomthetho borhulumente wephondo kwakunye neKomiti yoChongo yeMithetho neMiba yoMgaqo siSeko. Ngenxa yezi ndibano iNdlu yowiso-mthetho yePhondo leGauteng yatenxa kwisigqibo esathatythwa kwindibano yoluntu oko ikwenza ngokuthi ixhase uHlalutyo lweShumi elineSibini olubandakanya umasipala wase Merafong kwiPhondo loMntla Ntshona. Kwizindululo zokugqibela iNdlu yowiso-mthetho yaseGauteng yaphakamisa izizathu ngezizathu zoguqulo. ${ }^{30}$

Imibandela eyayi phambi kwenkundla yile: (i) ingaba lalukho ngokwaneleyo na uthatyatho nxaxheba luluntu olwenziwa yipalamente yephondo; (ii) ingaba isigqibo sokungabandakanyi uMasipala waseMerafong eGauteng (uguqulo lommiselo) sasifanelekile na.

29 Indlela umasipala waseMerafong wawuchazwe ngayo kumqulu weZaziso zePhondo loMntla Ntshona.

30 IKomiti yeMicimbi yeSebe loRhulumente boMasipalat-iGauteng

"Indawo iKomiti eme kuyo emva ko kuqhutywa kwengxoxo zeKomiti yoChongo yeBhunga laMaphondo leSizwe"

Xa ivitho yePhondo laseGauteng ibandakanya wonke uMthetho oHlalutywayo woMgaqo siSeko njengoko ibandakanya imida exananazileyo yomasipala, UMthetho woPhengululo woMasipala kuya kufuneka ukuba urhoxiswe ePalamente, kwaye unyulo lomasipala kofuneka lwenziwe phantsi kwezimiselo ezikhoyo zomasipala, oko kukuthi, kunye nomasipala abanemida exananazileyo.

Xa kunokuchazwa izindululo ngenkcazelo efinyeziweyo kwizindululo zoMthetho oHlalutywayo woMgaqo siSeko onokuvotelwa liphondo, imiphumela ifana naleyo yokungaphunyezwa koMthetho woHlalutywayo woMgaqo siSeko. Singafane senze ingxoxo sithi iGauteng inakho ukwala okanye ithi vitho elo solotya lisisiphakamiso kuShedyuli $1 \mathrm{~A}$ ethi inike inkcazelo yommandla; lo nto iya kuthetha ukuba ubunganga bokuba nemida exananazileyo iyarhoxiswa, ngelo lixa imida ekhoyo eGauteng iya kuhlala injalo. Iziphumo zoku iya kukuba uMmandla waseWest Rand kunye neTshwane, Ekurhuleni kunye neMetsweding iya kuthi ichaphazeleke. Aba masipala (kunye nomasipala besithili abachaphazelekayo) kuya kufuneka ukuba bahlakazwe kunye nezo ndawo zomasipala ekuxoxwa ngabo abathi babe phantsi kweGauteng. Ezo ndawo zinemida exananazileyo eziphantsi kwamanye amaphondo kuya kufuneka ukuba ziphinde zimiselwe imida ngokutsha kwaba masipala batsha.

Okona kuntsokotha kumandla kuya kuba koko kukhoyo lwemida aseGauteng ekusafuneka ukuba isekwe mayela nemimandla kamantyi engekasetyenziswa okanye ekungabhekiswanga kuyo kuHlalutyo loMthetho weShumi elineSibini loMgaqo siSeko. Ngaphaya koko, inguqulelo ezenziwayo kuMthetho oyilwayo oya kuba kuba yimfuneko kuHlalutyo loMgaqo siSeko ukuhlangabezana nendawo iGauteng ezifumana ikuyo, lo nto ise nokuba ngathi akusenako ukuba kungagqityezelwa umthetho oHlalutywayo oko kusenzelwa uvoto loMasipala beZithili,ngokoke, unyulo luya kuqhubeka phantsi kwezimiselo ezikhoyo." 


\subsection{Ukufaneleka kwengqiqo}

Umbandela woqiqo uquka indlela uluntu oluthi lubandakanywe ngayo ekwenzeni izigqibo kwiNdlu yowiso-mthetho yePhondo laseGauteng. Emva kokufumana ingcebiso zomthetho ipalamente yaguqula indlela ethi yenze ngayo izinto, ekuthini ipalamente yaxelelwa ukuba inokuvota ngokuthi "ivumelane okanye ingavumelani" njengoko ingenakho ukuba ingahlalutya ize iguqule umthetho oyilwayo. Umbuzo othi uphakame ngulowo ufuna ukuqonda ukuba ingaba ipalamente yenza ngengqiqo na xa yayingazange ibuye umva ize eluntwini ukuza kufuna ingcamango zabo emva kokuguquka kwezinto. UVan der Westhuizen iJaji, yesininzi, yafumanisa ukuba izigqibo zeNkundla zangaphambili ${ }^{31}$ azinyanzelisi thetha-thwano oluqhubekekayo, ukungabuyeli eluntwini kungafaniswa nokungabina mbeko kodwa akuna kukhokelela ekubeni isigqibo seNdlu yowiso-mthetho singabikho semthethweni. ${ }^{32}$ Izimvo zoluntu ziviwe kunye nezibhaliweyo, ngoko ke umsebenzi wokunika ithuba uwonke-wonke ukuba athabathe inxaxheba wenziwe ngokufanelekileyo. Ngoko ke iNkundla ayizange ifumane ubungqina bokuba iNdlu yowiso-mthetho yaseGauteng ayisebenzisanga amagunya ayo ngoku ngena ngqiqo.

AbaBheni nabo bavakalisa ukuba iNdlu yowiso-mthetho yaseGauteng ayizange ibeke izinto elubala ibe nokuguqulwa izimvo kuba umPhathiswa wathi kwantla-ndlolo wachaza esidlangalaleni kwi-website ukuba uMasipala waseMerafong uya kuthi abandakanywe phantsi kwePhondo loMntla Ntshona ${ }^{33}$ kwaye eso sigqibo senziwa phambi kokuba uluntu luthathe inxaxheba. Lento ukuba kwakukho ungoxinzelelo lwezopolitiko olwabangela ukuba leNdlu ibe madolo' nzima ekunikeni izizathu zokugqibela zeziphakamiso. ${ }^{34}$

INkundla yaphakamisa ukuba iziphumo zisekelezelwe kwizinto ezikhoyo kunye nobungqina obuphambi kwayo; ayikwazi kwenza sigwebo ekubeni kubekho kusinina uxinzelelo lwezopolitiko. ${ }^{35}$

\subsection{Ingqiqo}

Izizathu ezanikwa yiKomiti yeMicimbi yeSebe mayela nokujikwa kwegunya elalinalo sasisekelezelwe kwiingcebiso zomthetho ezafumaneka kubacebisi bomthetho bakarhulumente zokuba iphondo linokuthi liphumeze okanye likhabe lomthetho oyilwayo woMgaqo siSeko, oko likwenza phantsi kwecandelo 74(8) loMgaqo siSeko kwaye akukho luhlalutyo nanguqulo elinokuthi lwenzeke kwiBhunga lamaPhondo leSizwe. Ezi zizathu zikhomba imiphumela emibi ebinokuvela xa iNdlu yowiso-mthetho inoku ngavumelani nokupasiswa kwalemithetho ezi zezi: (i) Umthetho oyilwayo uwonke kwakufuneka urhoxiswe nto leyo ethetha ukuba unyulo lomasipala luya kuqhubeka phantsi komasipala abanemida exananazileyo; (ii) nokubangaba iNdlu yowiso-mthetho yaseGauteng ikhaba isolotya elithile elinento yokwenza

1 Doctors for Life International (fn 14 ngasentla); Matatiele 2 (fn 21 ngasentla).

Merafong (fn 11 ngasentla) umhlathi 60.

Merafong (fn 11 ngasentla) umhlathi 47.

Ibid.

Merafong (fn 10 ngasentla) umhlathi 48. 
nemida yaseGauteng, isiphumo soko siya kufana nokungathi "kupheliswe" abanye omasipala kwaye (iii) unyulo loMasipala bezithili luya kuchaphazeleka kuba oomasipala belizwe lonke baya kusoloko imiselwa phantsi kwezithili zeeNkundla zooMantyi. AbaBheni bazama ukucela umngeni ngokumayela nengqiqo iNdlu yowiso-mthetho yaseGauteng oko kusenziwa kwimiba emibini. Okokuqala, iNdlu yowiso-mthetho yaseGauteng yenza izinto ngaphandle kwengqiqo xa yayishiya kwesinomhlwa igunya enalo oko ikwenza kwiBhunga lamaPhondo leSizwe, yathi yavotela iMerafong ukuba ibe yinxalenye yePhondo loMntla Ntshona. ${ }^{36}$ INdlu yowiso-mthetho yaseGauteng yenza izigqibo ezingenangqiqo kuba ayizange iphumelele ukunikezela izizathu zokushiya kwesinomhlwa igunya layo kwiBhunga lamaPhondo leSizwe. ${ }^{37}$ Okwesibini, abaBheni baxoxa ukuba isigqibo ngokunyanisekileyo asinangqiqo kuba ufakelo lweMerafong kwiPhondo loMntla Ntshona lachaphazela kakubi ubomi boluntu lwaseMerafong. ${ }^{38}$

Uvavanyo lwengqiqo kukuba ingaba ubunganga boluntu bubandakanywa kunye nemisebenzi yoburhulumente obufanelekileyo. ${ }^{39}$ INkundla yagqiba kwelokuba, isigqibo seNdlu yowiso-mthetho yaseGauteng sasiyinxalenye yenjongo eyiyo yokuphelisa omasipala abanemida exananazileyo kunye nokwenza omasipala abahlumileyo kwezoqoqosho ${ }^{40}$ kwaye ngoko ke sibenengqiqo. INkundla yaqaphela kananjalo ukuba ngokwe nkqubo yokwahlulwa kwamandla okulawula, iinkundla azivumelekanga ukuba ziguqule izigqibo zikarhulumente ukuba zisemthethweni nokuba iinkundla zingavumelani nezozigqibo. ${ }^{41}$ Ngokubhekiselele kwisininzi ubunganga bokuqulunqa uthatyatho nxaxheba luluntu abuthethi ukuba izimvo zoluntu ziya kuba zeziphumelelayo. ${ }^{42}$ Isigwebo sidlulelayo sithi urhulumente akanakho ukulindeleka ukuba abopheleleke kwimfuno zabantu abambalwa, kwaye uthatyatho nxaxheba luluntu lumele ukuhlangabezana nolonyulo kunye nolawulo lwesininzi, kwaye "lungaphixani okanye lungananzi okanye lubanqande". ${ }^{43}$

\section{INGXOXO}

Izizathu ezithi zisetyenziswe xa kujongwa ingqiqo zezi: (i) ukubaluleka komthetho oza kuphunyezwa, ulandelwa yi (ii) igalelo umthetho oya kuba nalo kuluntu. Kunzima ukuqonda ukuba kutheni iNkundla yafumanisa ukuba akuyomfuneko kwipalamente yephondo ukuba ibuyele kuluntu lwaseMerafong xa ngaba iimeko ebe kudityanwa ngazo ziye zaguqa (ezo izingcebiso ezivela kwiingcali zomthetho). Ukuguqulwa kwemida kuye kwaba nemiphumela engemihle kumalungelo abalulekileyo oluntu ${ }^{44}$, njengoko kuxoxwa

\footnotetext{
36 Merafong (fn 10 ngasentla ) umhlathi 66.

37 Ibid.

38 Ibid.

39 Merafong (fn 10 ngasentla ) umhlathi 63.

40 Merafong (fn 10 ngasentla ) umhlathi 96.

41 Merafong (fn 10 ngasentla ) umhlathi 63.

42 Merafong (fn 10 ngasentla ) umhlathi 50.

43 Merafong (fn 10 ngasentla ) umhlathi 26.

44 Amalungelo abalulwe kumacandelo 21 (3) ne 10 oMgaqo siSeko.
} 
ngu-O' Regan iJaji kwityala leMatatiele $1^{45}$, xa abantu abachaphazeleka ngqo bengabandakanywa ngokukuko kwinkqubo yokwenziwa kwemithetho.

Ukungaphumeleli ukwenza ingxelo kuluntu lwaseMerafong, ngokubhekiselele kwisigqibo sesininzi seNkundla alufikeleli kwinqanaba lokungabi nangqiqo nto leyo ibi nokukhokelela ekubeni uMthetho woGuqulo loMgaqo siSeko weShumi elineSibini ungabi nakwamkeleka. Ukususela kwizigwebo zangaphambili ${ }^{46}$ uxanduva lokwenza uthatyatho nxaxheba luluntu luchazwe njengelona libalulekileyo kwidemokhrasi yoMgaqo siSeko. USachs iJaji wathi "kweli lizwe lethu uthatyatho nxaxheba luluntu ngalo lonke ixesha luxanduva lorhulumente osekelezelwe kuMgaqo siSeko ngokusemthethweni" ${ }^{47}$, ngoko ke, apho isebe lowiso-mthetho lisenza isigqibo, eso sigqibo singabonakalisi ngokufanelekileyo oko kwakuthe kwafunyanwa ngelixa kusenziwa uthatyatho nxaxheba noluntu, ukwamkeleka komthetho ophuma kweso sigqibo uthi ube ngulowo unesiphene. KwiDoctors for Life iNkundla yasekelezela ukuba xa umthetho uye waphunyezwa ngendlela efanelekileyo, uluntu ngokubanzi luya kuwamkela umthetho onjalo. ${ }^{48}$ Kwisigwebo sesininzi seMerafong iNkundla yaphawula yathi:

"Abezopolitiko, ababonakala bengabahloniphi abavoti babo, okanye abangazifeziyo izithembiso zabo ngaphandle kwengcaciso, kumele benziwe bamele izenzo zabo. Inkqubo yedemokhrasi iveza zonke iinkalo ngokuphathalele koku, enye yazo kukuthi kubanjwe ulonyulo rhoqo". ${ }^{49}$

Liyinyani eli kodwa xa inxanxheba enikwa uluntu ingena ntsingiselo, unyulo ingaba kukuphela kwesisombululo uluntu olunaso. Leyo imbono iphikisana mpela nenjongo yokunika uluntu inxaxheba kwimisebenzi yowiso-mthetho, kaloku oku kuthintela imeko apho uluntu lubenezwi kurhulumente kanye eminyakeni emihlanu. Indima yenxaxheba yoluntu sisiqinisekiso sobukho boburhulumente "obunemfezeko, obuzenzo ziselubala, obunentembeko". ${ }^{50}$

\section{ISIPHELO}

Amandla eNkundla yoMgaqo siSeko axhomekeke ekukwazini ukukhusela isidima sabo bambalwa ngokwenani kunye nabo basesichengeni. Imeko yokuba uvavanyo lwengqiqo kuxanduva lokuqulunqa uthatyatho nxaxheba luluntu lungenazo izikhuseli51 oko kushiya ipalamente inobunganga bokwenza umnyingi nje oqulunqwe luqiqo kunye novavanyo lwengqiqo. Inkqubo yomnyingi kubandakanyo loluntu ibangele umda omkhulu onokuthi uvulele umthetho obalulekileyo oya kuthi ube negalelo kuluntu oya kuthi uphunyezwe njengowona uqiqiweyo.

\footnotetext{
45 UMasipala waseMatatiele kunye naBanye v UMongameli woMzantsi Afrika kunye naBanye (1) 2006 (5) BCLR 622 (CC) Matatiele 1.

46 Doctors for Life International (fn 14 ngasentla); Matatiele 2 (fn 21 ngasentla).

47 Doctors for Life International (fn 14 ngasentla) umhlathi 231.

48 Doctors for Life International (fn 14 ngasentla) umhlathi 205.

49 Merafong (fn 10 ngasentla ) umhlathi 60.

50 Icandelo 41(1)(c) kuMgaqo-siseko.

51 Imigaqo enokuthi ikhusele uthatyatho nxaxheba yayike yavezwa kuKing naBanye $v$ Fidelity Fund Board of Control naBanye 2006 (4) BCLR 462 (SCA).
} 
Ukumisela inqanaba eliphantsi kangako oko kuthetha ukuba ugxekoncomo lomthetho kumanye amasebe karhulumente alunaziphumo zizizo. USachs J, wayekhe walumkisa ngelithi ingaba lihlazo ukuba umsebenzi wokunika uluntu inxaxheba ngumboniso nje ongena ntsingiselo wathi:

"Kuya kuba akufanelekanga ukucebisa ukuba ingqwalasela kwiNldu yoQulunqo Mgaqo siSeko iyalukhuthaza uthatyatho nxaxheba koluntu kubunjo lwemithetho, oko kwaba ngumfuziselo nje ongabheke phi mayela nomgaqo siseko ngakwicala layo". ${ }^{52}$

Uluntu, ngokubhekiselele kwisininzi, luyakuba nenxaxheba ethe vetshe kulonyulo oluzayo. ${ }^{53}$

\section{ULUHLU LWEENCWADI}

Buccus, I 'Towards developing a public participation strategy for South Africa's Provincial Legislatures (2006) Special Focus: Centre for Public Participation

Ingxelo yonyaka yeSebe Locwangciso no Rhulumente woMmandla yephondo laseGauteng 2000 / 2001

Imvumelwano emalunga nolawulo koomasipala abamida exananazileyo eyayilwa kwiSiqheba sabaQuquzeleli baka Momgameli ngomhla we 1 Novemba 2002

Motala, Z kunye noRamaphosa, C 'Constitutional Law: Analysis and cases' Oxford University Press (2002) 9

Iziphakamiso mayela nokuguqulwa kwemida - Isaziso sikaRhulumente 2818931 Oktobha 2005

Sikhakhane, M 'In re: Doctors for Life case and Matatiele case' Intlangano yeSAHRC/SAIFAC kushukuxwa izigqibo zeNkundla yoMgaqo siSeko

Doctors for Life International (fn 13 ngasentla) umhlathi 227.

3 Merafong (fn 10 ngasentla ) umhlathi 60. 